Nonlin. Processes Geophys., 21, 41-47, 2014

www.nonlin-processes-geophys.net/21/41/2014/

doi:10.5194/npg-21-41-2014

(c) Author(s) 2014. CC Attribution 3.0 License.

(c) (i)

\title{
Four-dimensional energy spectrum for space-time structure of plasma turbulence
}

\author{
Y. Narita ${ }^{1,2}$ \\ ${ }^{1}$ Space Research Institute, Austrian Academy of Sciences, Schmiedlstr. 6, 8042 Graz, Austria \\ ${ }^{2}$ Institut für Geophysik und extraterrestrische Physik, Technische Universität Braunschweig, 38106 Braunschweig, Germany \\ Correspondence to: Y. Narita (yasuhito.narita@oeaw.ac.at)
}

Received: 15 May 2013 - Revised: 15 December 2013 - Accepted: 25 December 2013 - Published: 9 January 2014

\begin{abstract}
A parametric model of the inertial-range energy spectrum is constructed for plasma turbulence in the fourdimensional wave vector and frequency domain. The model is based on that of the Eulerian wavenumber-frequency spectrum developed for describing fluid turbulence, and includes wave vector anisotropies in the three-dimensional wave vector domain by approximating the spectrum to a set of ellipses. The shape of the four-dimensional spectrum is determined by the Doppler shift, the Doppler broadening, and anisotropy coefficients. The model is applied to the magnetic energy spectrum in the near-Earth solar wind measured by four Cluster spacecraft, and the set of the spectral parameters are determined observationally. In this way, space-time structure of plasma turbulence can be condensed into a small number of parameters, which is suitable for evaluating the energy spectra in observational and numerical studies on the quantitative basis.
\end{abstract}

\section{Introduction}

Plasma turbulence appears in various astrophysical systems and plays an important role as an effective transport mechanism of mass, energy, and angular momentum. It can be found in the Sun and the heliosphere (Petrosyan et al., 2010; Bruno and Carbone, 2013), planetary magnetospheres (Zimbardo et al., 2010), accretion disks (Balbus, 2003; Julien and Knobloch, 2010), and interstellar medium (Elmegreen and Scalo, 2004; Scalo and Elmegreen, 2004). Above all, solar wind is particularly interesting, since it is the only fully developed plasma turbulence accessible to us, allowing detailed investigations using spacecraft in situ in space (Tu and Marsch, 1995; Bruno and Carbone, 2013). One of the re- cent achievements of multi-spacecraft mission Cluster (Escoubet et al., 2001) is the fact that spatial and temporal variations can be distinguished in the measurements. Furthermore, three-dimensional spatial structure can also be determined using multi-spacecraft data. Turbulence is fundamentally a spatio-temporal phenomenon, and exhibits an interplay of spatial and temporal effects (Carbone et al., 2011; Servidio et al., 2011). The large-scale flow causes Doppler shift and broadening in the measurements, and furthermore, the presence of the large-scale magnetic field imposes anisotropy in the energy spectrum. It is a challenging task both in theory and experiment to reveal the energy spectrum of plasma turbulence in the four-dimensional Fourier space spanned by frequencies and wave vectors.

There is a growing amount of evidence that the energy spectrum of plasma turbulence is intrinsically anisotropic in the wave vector domain. Earlier studies on the quasiperpendicular wave vectors with respect to the large-scale magnetic field (Matthaeus et al., 1990; Bieber et al., 1996) have been extended to various spatial scales using numerical simulations: magnetohydrodynamic scales (Matthaeus et al., 1996), ion kinetic scales using hybrid plasma simulation (Verscharen et al., 2012; Comişel et al., 2013) or gyro-kinetic treatment (Howes et al., 2011), and electron kinetic scales using particle-in-cell simulations (Camporeale and Burgess, 2011; Gary et al., 2012; Chang et al., 2013). Vlasov-Maxwell hybrid simulation study provides evidence of electric power anisotropy (Valentini et al., 2010). Recent studies using Cluster data not only support the picture of turbulent fluctuations associated with quasi-perpendicular wave vectors, but also suggest axial asymmetry with respect to the directions around the large-scale magnetic field (Turner et al., 2011). 
In this manuscript, I propose a parametric method to characterize the four-dimensional (4-D) energy spectrum of plasma turbulence using a model spectrum and observational data from Cluster in the solar wind. The model is given as analytical and contains five free parameters that need to be determined by the observations. Using the free parameters obtained by a fitting procedure, the four-dimensional energy spectrum can be reconstructed as an ideal one. Such a data-driven reconstruction has become possible by the development of the novel high-resolution spectral estimator, the MSR technique (Multi-point Signal Resonator) (Narita et al., 2011a). Complementary approach is to produce plasma turbulence in numerical simulations and to analyze the spectrum using the model. While ideal situations can easily be set in the simulations (e.g., constant mean field, choice of fluctuation amplitude relative to the mean field, and the values of plasma parameter beta), the numerical method necessarily depends on the choice of the plasma model. The energy spectrum model proposed in this manuscript assumes that the hydrodynamic treatment of frequency dependence is valid in that the effect of finite wave propagation speed or dispersion relation is negligible.

\section{Model construction}

The model of the 4-D energy spectrum is constructed as a generalization of the inertial-range spectrum for fluid turbulence recently derived by Wilczek and Narita (2012). In essence, the fluid turbulence model makes use of the random sweeping hypothesis developed by Kraichnan (1964), and can reproduce the elliptic model of two-time, two-point correlation function of turbulent flows (He and Zhang, 2006; Zhao and He, 2009). The model spectrum is expressed as a product of the three-dimensional (3-D) energy spectrum in the wave vector domain $E(\boldsymbol{k})$ and the Gaussian frequency distribution $F(\boldsymbol{k}, \omega)$ as

$$
\begin{aligned}
E(\boldsymbol{k}, \omega) & =E(\boldsymbol{k}) F(\boldsymbol{k}, \omega) \\
F(\boldsymbol{k}, \omega) & =\frac{1}{\sqrt{2 \pi k^{2} V^{2}}} \exp \left[-\frac{(\omega-\boldsymbol{k} \cdot \boldsymbol{U})^{2}}{2 k^{2} V^{2}}\right] \\
E(\boldsymbol{k}) & =E_{0}|\kappa(\boldsymbol{k})|^{-11 / 3} \\
\kappa^{2}(\boldsymbol{k}) & =a_{\|}^{2} k_{\|}^{2}+a_{\perp 1}^{2} k_{\perp 1}^{2}+a_{\perp 2}^{2} k_{\perp 2}^{2} .
\end{aligned}
$$

The spectrum is defined in the 4-D Fourier space spanned by the Eulerian (angular) frequency $\omega$ and the wave vector $\boldsymbol{k}$. The Gaussian frequency distribution is centered at the Doppler relation $\omega=\boldsymbol{k} \cdot \boldsymbol{U}$, and has the standard deviation $k V$. In this approach, the spatial structures are assumed to be swept by the mean flow $\boldsymbol{U}$ and the random sweeping flow $V$. The former causes the Doppler shift, and the latter causes the Doppler broadening. It is worth mentioning that the notion of the Doppler shift and broadening is valid not only in high-frequency phenomena (sound waves, electromagnetic waves), but also in low-frequency plasma turbu- lence. The 3-D energy spectrum $E(\boldsymbol{k})$ is modeled as a set of ellipses determined by three coefficients $\left(a_{\|}, a_{\perp 1}\right.$, and $\left.a_{\perp 2}\right)$. The set of the coefficients quantifies wave vector anisotropy between the parallel and the perpendicular directions to the large-scale magnetic field by the ratios $a_{\perp 1} / a_{\|}$or $a_{\perp 2} / a_{\|}$and axial asymmetry with respect to the directions around the large-scale field by the ratio $a_{\perp 2} / a_{\perp 1}$. We use the large-scale magnetic field (in the direction $\boldsymbol{e}_{\text {mag }}$ ) and the mean flow direction $\left(\boldsymbol{e}_{\text {flow }}\right)$ to establish the coordinate system in the wave vector domain. Three basis vectors are mutually orthogonal and determined by the following relations.

$$
\begin{aligned}
& \boldsymbol{e}_{\perp 1}=\left(\boldsymbol{e}_{\mathrm{flow}} \times \boldsymbol{e}_{\mathrm{mag}}\right) \times \boldsymbol{e}_{\mathrm{mag}} \\
& \boldsymbol{e}_{\perp 2}=\boldsymbol{e}_{\mathrm{flow}} \times \boldsymbol{e}_{\mathrm{mag}} \\
& \boldsymbol{e}_{\|}=\boldsymbol{e}_{\mathrm{mag}}
\end{aligned}
$$

The perpendicular-1 direction is closest to the mean flow direction in the plane perpendicular to the large-scale magnetic field.

The model spectrum (Eq. 1-4) represents the simplest extension of the fluid turbulence spectrum in that the minimum set of free parameters is used. The model spectrum is two-sided, viz., it is symmetric with respect to sign reversal of frequencies and wave vectors $(\omega \rightarrow-\omega$ and $\boldsymbol{k} \rightarrow$ $\boldsymbol{- k}$ ). The spectral energy is scaled to $E_{0}$, and inherits the power-law index of Kolmogorov's 3-D inertial-range spectrum (Kolmogorov, 1941), such that the isotropic limit $\left(a_{\|} \rightarrow\right.$ $1, a_{\perp 1} \rightarrow 1$, and $\left.a_{\perp 2} \rightarrow 1\right)$ restores the inertial-range spectrum of fluid turbulence. One of the anisotropy coefficients may be set to unity by redefining the scale factor $E_{0}$ and the two other coefficients. In this work, $a_{\|}$is set to unity.

The use of the Gaussian frequency distribution is based on the approximation that small-scale fully-developed turbulence is swept by the large-scale flow in an advective fashion. The mean flow causes Doppler shift, while the variation of the large-scale flow causes Doppler broadening around the Doppler shifted frequency (Fig. 1). In the limit of vanishing Doppler broadening $(V \rightarrow 0)$ the Gaussian frequency distribution reduces to the Dirac delta function $F(\boldsymbol{k}, \omega) \propto$ $\delta(\omega-\boldsymbol{k} \cdot \boldsymbol{U})$, restoring Taylor's frozen-in flow hypothesis (Taylor, 1938). The symbol $V$ is independently defined as a free parameter in the model. The notion of the large-scale flow is valid as far as the fluctuations is regarded as ideally convected structures. Specifically, the flow (or the plasma) should not evolve intrinsically within the measured time interval, and this can be examined, e.g., by looking for the existence of dispersion relations that deviate from the Doppler relation in the frequency-wavenumber diagram. Therefore, the model spectrum neglects the wave propagation effect such as Alfvén waves, and the model is more suited for applying to super-Alfvénic flows. 


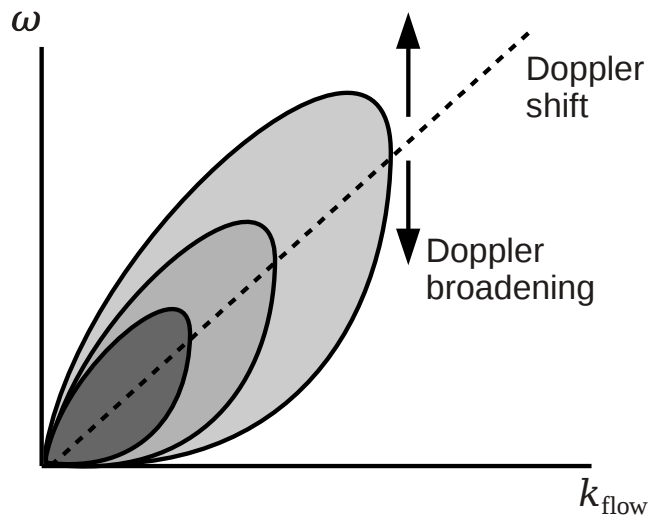

Fig. 1. Sketch of the inertial-range spectrum in the wavenumberfrequency domain. The wavenumber is aligned with the mean flow direction.

\section{Application to solar wind turbulence}

The model spectrum is used as an analysis tool to characterize the space-time structure of solar wind turbulence. By doing so, it is possible to condense the turbulence spectrum into only several free parameters. Cluster spacecraft are most useful for such a task, as the 4-D energy spectrum can be estimated directly from the four-point magnetic field data (Balogh et al., 2001) by incorporating the MSR analysis technique. The data are taken on 12 February 2005, 06:3008:00 UT, for the analysis. This interval was selected under the following criteria:

1. Cluster formed a nearly regular tetrahedron which is suitable for wave vector analysis. The tetrahedral quality parameter $Q_{G}$ (von Stein et al., 1992) in the range 2.9-3.0 was set. This parameter quantifies the tetrahedral configuration as a fractal dimension.

2. Inter-spacecraft separation was about $1000 \mathrm{~km}$ and larger than the thermal ion gyro-radius (of the order of $100 \mathrm{~km}$ ) in the solar wind. Turbulence may be regarded as magnetohydrodynamic on that scale, and kinetic wave dispersion effect can be neglected.

3. Fluctuations of the magnetic field and the plasma are quasi-stationary in the sense that the large-scale field may be regarded as nearly constant (cf. the relative fluctuation amplitude to the large-scale field is about $10.2 \%)$.

4. Frequency spectra for the magnetic field fluctuations (at all four spacecraft) exhibit a power-law with the index $-5 / 3$. Fig. 2 displays the time series of the magnetic field magnitude, the ion bulk speed, and the ion number density (Rème et al., 2001) obtained by Cluster-1.

The mean values are evaluated in the temporal domain, and are $2.5 \mathrm{nT}$ (magnetic field), $548.9 \mathrm{~km} \mathrm{~s}^{-1}$ (ion bulk speed),

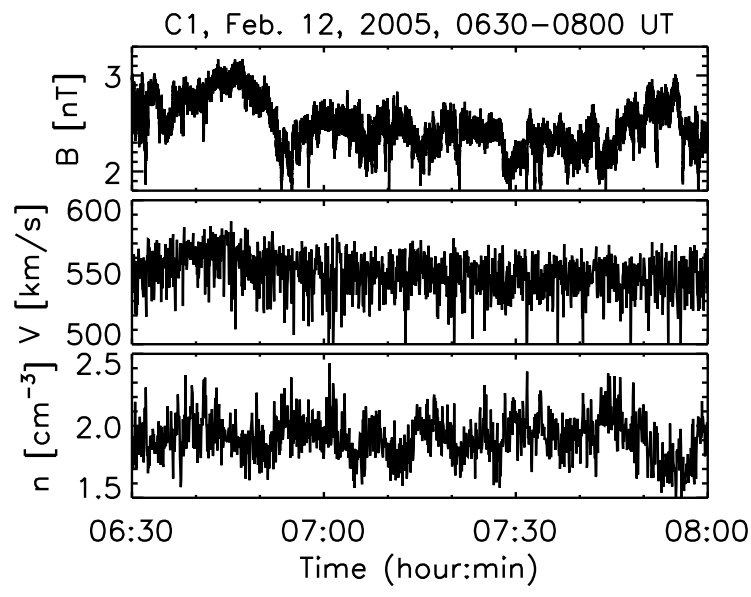

Fig. 2. Time series plot of the magnetic field magnitude, the ion bulk speed, and the ion number density measured by Cluster-1 in the solar wind.

and $1.9 \mathrm{~cm}^{-3}$ (ion number density). The Alfvén Mach number is about 13.7 on this time interval, and the plasma parameter beta is estimated to be about 5.4 using ion data. This interval represents a turbulent flow in a high-beta plasma.

The 4-D energy spectrum was estimated from the Cluster magnetic field data (measured by the fluxgate magnetometer) first by Fourier transforming the four-point time series data into the frequency domain (using the Fast Fourier Transform algorithm) and then by projecting the data into the 3-D wave vector domain using the MSR technique. This method was developed particularly for studying waves and turbulence using four-point magnetic field data, and makes extensive use of the 12-by-12 covariance matrix (three components of the magnetic field measured at four spacecraft) by combining the least-square projection (known as the wave telescope or $k$ filtering technique) and the eigenvalue analysis of the covariant matrix, known as the MUSIC algorithm (Multiple Signal Classification) (Schmidt, 1986). The MSR technique is based on the assumption that the measured fluctuations represent a set of plane waves and that the fluctuations contain smallamplitude isotropic noise. The energy spectrum is given as a function of spacecraft-frame frequencies and wave vectors. The frequency range up to $1.2 \mathrm{rad} \mathrm{s}^{-1}$ and the wavenumber range up to $0.0023 \mathrm{rad} \mathrm{km}^{-1}$ are used to determine the free parameters of the model spectrum. The upper limit of the frequency range to analyze is the spacecraft separation $\left(k_{\max }\right)$, which yields, using a given relationship $\omega \propto k$, the corresponding frequency $\omega_{\max }$ that allows one to avoid the spatial aliasing effect (Narita and Glassmeier, 2009; Sahraoui et al., 2010). Here, Taylor's hypothesis gives a reasonable estimate to set the frequency range in the analysis.

A fitting procedure is applied to the measured spectrum in two distinct domains. One is the plane spanned by the streamwise wavenumbers and the frequencies, and the other is the 3-D wave vector domain after integrating the 4-D 

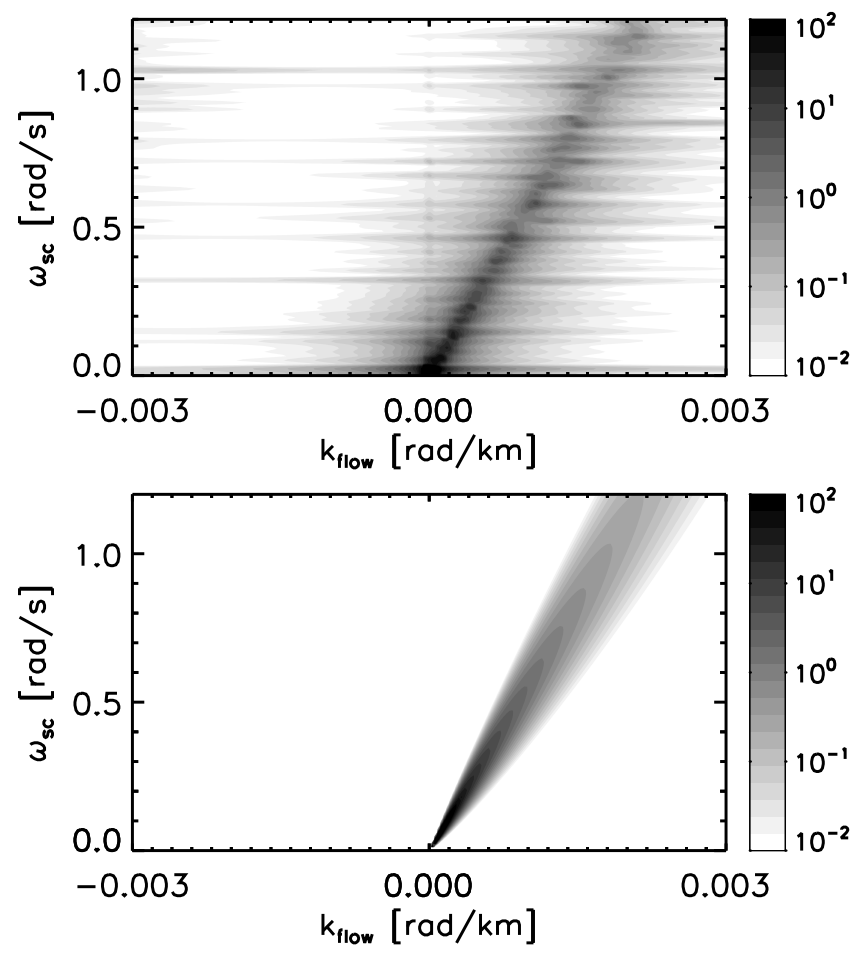

Fig. 3. Slice of the energy spectrum in the plane spanned by spacecraft-frame frequencies and streamwise wavenumbers derived by four Cluster spacecraft data (upper panel) and the reconstructed energy spectrum using the method of model fitting (lower panel). Best fitting was obtained for the parameter set $U=547.6 \mathrm{~km}$ (Doppler shift) and $V=49.6 \mathrm{~km}$ (Doppler broadening).

spectrum over the frequencies. The fitting procedure in the former domain determines the Doppler shift $U$ and the broadening $V$, while the fitting in the latter domain determines the anisotropy coefficients. Fig. 3 displays the measured spectrum and the reconstructed spectrum using the set of the best-fitting parameters $\left(U=547.6 \mathrm{~km} \mathrm{~s}^{-1}, V=\right.$ $\left.49.6 \mathrm{~km} \mathrm{~s}^{-1}\right)$. The time series data of the ion bulk speed from Cluster-1 yields the standard deviation $\sqrt{\left\langle|\delta \boldsymbol{v}|^{2}\right\rangle}=$ $20.0 \mathrm{~km} \mathrm{~s}^{-1}$ in the temporal domain. The Doppler shift estimated in the spectral analysis agrees with the ion bulk speed within the accuracy better than $1 \%$. The estimated Doppler broadening agrees with the ion bulk speed fluctuation only moderately, i.e., the broadening $V$ is larger than that estimated from the ion measurements by the factor of about 2.5 . The Alfvén speed estimated from the mean magnetic field and the ion number density is about $38.9 \mathrm{~km} \mathrm{~s}^{-1}$, which is markedly close to the broadening $V$.

The reconstructed spectrum shows a difference from the measurement in that the conic shape is not clearly visible in the data. Possible explanations are (1) insufficient scale separation, and (2) finite wave propagation speed. In the former scenario, since the model spectrum assumes that fluctuations consist of large-scale and small-scale fields, the dif-

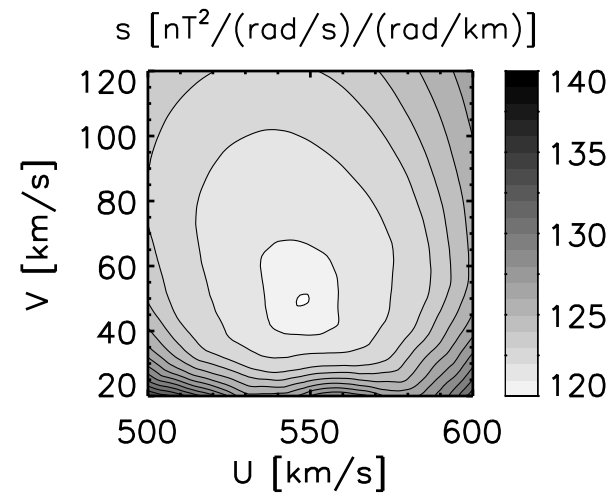

Fig. 4. Deviation between the measured spectrum and the model spectrum in the parameter space spanned by $U$ (Doppler shift) and $V$ (Doppler broadening).

Table 1. Key spectral shape parameters obtained from the Cluster data analysis for solar wind turbulence.

\begin{tabular}{cccc}
\hline$U$ & $V$ & $a_{\perp 1} / a_{\|}$ & $a_{\perp 2} / a_{\|}$ \\
\hline $547.6 \mathrm{~km} \mathrm{~s}^{-1}$ & $49.6 \mathrm{~km} \mathrm{~s}^{-1}$ & 0.73 & 0.31 \\
\hline
\end{tabular}

ference in the frequency dependence can be interpreted as a sign of insufficient scale separation. That is, the frequency broadening is no longer expressed by a constant Doppler broadening $V=$ const, but is a function of the wavenumber such as $V=f(k)$. In the present study, the parameters $U$ and $V$ are regarded as a constant, and these values are obtained by a least square method in the wavenumberfrequency domain (Fig. 4). In the latter scenario, since the model spectrum assumes that the frequency dependence represents solely Doppler shift and broadening and neglects the effect of finite wave phase speed, the difference in the spectrum may be interpreted as a sign of the existence of dispersion relations associated with, e.g., Alfvén waves, magnetosonic waves, or even dispersive waves. The intrinsic noise originating in the analysis technique is an unlikely source, since the MSR technique has been developed particularly to improve the signal-to-noise ratio of the conventional wave telescope technique significantly by a factor of about $10^{5}$ in the spectrum as shown in Fig. 3 in Narita et al. (2011a) by incorporating the eigenvalue analysis into the wave telescope technique. The intrinsic noise associated with the analysis technique is negligible.

The energy spectrum in the 3-D wave vector domain was determined by integrating the 4-D spectrum over the spacecraft-frame frequencies. The anisotropy shape parameters $\left(a_{\|}, a_{\perp 1}\right.$, and $\left.a_{\perp 2}\right)$ are evaluated in the three slices of the 3-D spectrum (planes at $k_{\|}=0, k_{\perp 1}=0$, and $k_{\perp 2}=0$ ) with the help of the anisotropy angle $\theta_{i j}$ in the plane spanned by $k_{i}$ and $k_{j}$ (the indices $i$ and $j$ denote the three components of the wave vector). The anisotropy angle was introduced by 

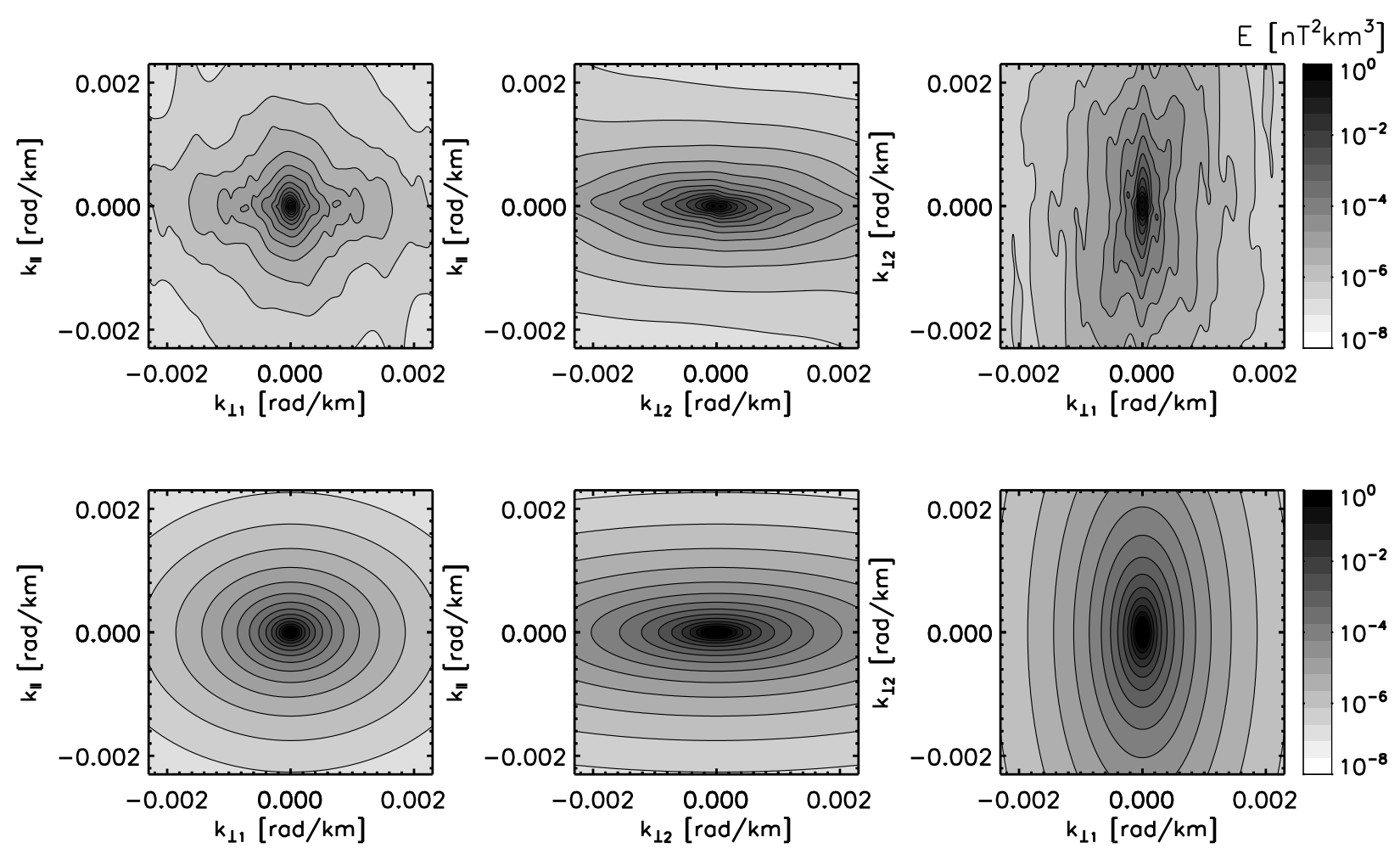

Fig. 5. Slices of the energy spectrum in the 3-D wave vector domain using Cluster spacecraft data in the solar wind (upper panels), and its reconstruction using the model spectrum (lower panels).

Shebalin et al. (1983) and its adapted form was used in the present analysis as

$\tan ^{2} \theta_{i j}=\frac{\sum_{k} k_{j}^{2} E\left(k_{i}, k_{j}\right)}{\sum_{k} k_{i}^{2} E\left(k_{i}, k_{j}\right)}$

The summation runs over all the wave vector components in the plane of interest. Fitting procedure using the anisotropy angle yields the set of optimal ratios of the anisotropy coefficients, $a_{\perp 1} / a_{\|}=0.73$, and $a_{\perp 2} / a_{\|}=0.31$. Fig. 5 displays the measured spectrum (upper panels) and the reconstructed spectrum using the best-fit parameters (lower panels).

In the $k_{\|}-k_{\perp 1}$ plane, the spectrum is not elliptic but exhibits two different kinds of anisotropy. At lower wavenumbers (below $0.0008 \mathrm{rad} \mathrm{km}^{-1}$ ) the spectrum extends in the parallel direction, while at higher wavenumbers the extension turns to the perpendicular direction. This transition cannot be reproduced in the model spectrum, and the reconstructed spectrum is only moderately anisotropic. In the $k_{\|}-k_{\perp 2}$ plane, the spectrum is clearly anisotropic, elongated in the perpendicular direction. Approximation to ellipses can reproduce the spectral contours reasonably. In the $k_{\perp 1}-k_{\perp 2}$ plane, the spectrum is axi-asymmetric with detailed structures. The spectrum is elongated in the direction perpendicular to the mean flow, i.e., the spectral extension is in the direction of interplanetary electric field. These results support earlier studies using single and multiple spacecraft measurements that so- lar wind turbulence is anisotropic with respect to the wave vectors, and is axi-asymmetric around the direction of the large-scale magnetic field (Narita et al., 2010, 2011b; Chen et al., 2012).

\section{Conclusion and discussion}

The model energy spectrum is constructed in a simple fashion using analytic expression. The model is based on various assumptions such as incorporation of the random sweeping hypothesis, no wave propagation (or no dispersion relation), and elliptic approximation of the wave vector anisotropy. Still, the model has the potential to condense the energy spectrum of plasma turbulence (and hence space-time variation of turbulence) into a set of handful free parameters. Six parameters need to be determined in reconstructing the 4-D energy spectrum, but the shape of the spectrum itself requires four parameters only: the Doppler shift $(U)$, the Doppler broadening $(V)$, and the anisotropy ratios $a_{\perp 1} / a_{\|}$ and $a_{\perp 2} / a_{\|}$. The ratio $a_{\perp 2} / a_{\perp 1}$ reflects the axial asymmetry of the spectrum around the directions of the large-scale magnetic field. This key parameter set derived from the Cluster data in the solar wind is summarized in Table 1.

There are strengths and weaknesses in the model spectrum. Since the model reduces the energy spectrum into a set of free parameters, various realizations of plasma turbulence 
can be compared more easily and systematically. For example, the model can be applied to studies of beta dependence of plasma turbulence. Evolution of plasma turbulence can be studied by tracking time dependence of the parameter set. Quantitative comparison with numerically produced turbulence is possible. The model spectrum is constructed in the Eulerian frame of reference, and does not require the correction for the Doppler shift (instead, the concept of Doppler broadening has been introduced), which saves the computation time in data analysis. On the other hand, the assumptions used in the model are limiting the applicability, and the conditions of the plasma and magnetic field need to be carefully examined. The measured spectrum in the $k_{\|}-k_{\perp 1}$ plane exhibits two different kinds of anisotropy (parallel and perpendicular spectral extensions), which prefers the twocomponent picture plasma turbulence describing a superposition of fluctuations associated with the parallel wave vectors and that associated with the perpendicular wave vectors (Matthaeus and Ghosh, 1999). Highly structured wave vector anisotropy cannot be resolved or reproduced in the model spectrum. Also, the choice of the basis vectors needs to be exercised, as the axial asymmetry in the measured spectrum may not always be associated with the direction of the mean flow or the direction of interplanetary electric field.

The axial asymmetry in the $k_{\perp 1}-k_{\perp 2}$ plane is found to be stronger than anisotropy in the $k_{\|}-k_{\perp 1}$ on the analyzed time interval, and weaker than that in the $k_{\|}-k_{\perp 2}$. This feature was already found in the other case study using Cluster data (Narita et al., 2011b). The strong axial asymmetry cannot be explained by the spectral deformation induced by the irregular tetrahedral configuration, as spacecraft formed a nearly regular tetrahedron during the measurements. Possible origins of the axial asymmetry include the effect of radial expansion of the flow, the effect of interplanetary electric field, and the coronal field structure. The model spectrum can be extended in various ways to reconstruct the measured spectrum more accurately. For example, the parameter $V$ may be treated as a function of the wavenumber. Another approach is to incorporate dispersion relations into the frequency dependence, which, however, requires the knowledge of the wave modes. Also, the presented model can be extended to describing the energy spectrum in the ion kinetic regime. For example, Comişel et al. (2013) introduced the Gaussian frequency distribution to measure the strength of sideband waves. The Doppler relation was also replaced by dispersion relations of linear modes assuming that linear modes co-exist in turbulent fluctuations. To conclude, model construction is an important task in plasma turbulence studies, and a suitable model will serve as a bridge connecting experimentally derived spectra and theoretical ones.

Acknowledgements. The research leading to these results has received funding from the European Community's Seventh Framework Programme under grant agreement 313038/STORM. The work conducted in Braunschweig is supported by Collaborative Research Center 963, Astrophysical Flow, Instabilities, and Turbulence of the German Science Foundation.

Edited by: A. C. L. Chian

Revied by: two anonymous referees

\section{References}

Balbus, S. A.: Enhanced angular momentum transport in accretion disks, Ann. Rev. Astron. Astrophys. 41, 555-597, doi:10.1146/annurev.astro.41.081401.155207, 2003.

Balogh, A., Carr, C. M., Acuña, M. H., Dunlop, M. W., Beek, T. J., Brown, P., Fornaçon, K.-H., Georgescu, E., Glassmeier, K.-H., Harris, J., Musmann, G., Oddy, T., and Schwingenschuh, K.: The Cluster magnetic field investigation: overview of in-flight performance and initial results, Ann. Geophys., 19, 1207-1217, doi:10.5194/angeo-19-1207-2001, 2001.

Bieber, J. W., Wannger, W., and Matthaeus, W. H.: Dominant two-dimensional solar wind turbulence with implications for cosmic ray transport, J. Geophys. Res., 101, 2511-2522, doi:10.1029/95JA02588, 1996.

Bruno, R. and Carbone, V.: The solar wind as a turbulence laboratory, Living Rev. Solar Phys., 10, 2, doi:10.12942/lrsp-2013-2, 2013.

Camporeale, E. and Burgess, D.: The dissipation of solar wind turbulent fluctuations at electron scales, Astrophys. J., 730, 114, doi:10.1088/0004-637X/730/2/114, 2011 (Correction, 735, 67, doi:10.1088/0004-637X/735/1/67, 2011).

Carbone, F., Sorriso-Valvo, L., Versace, C., Strangi, G., and Bartolino, R.: Anisotropy of spatiotemporal decorrelation in electrohydrodynamic turbulence, Phys. Rev. Lett., 106, 114502, doi:10.1103/PhysRevLett.106.114502, 2011.

Chang, O., Gary, S. P., and Wang, J.: Whistler turbulence at variable electron beta: Three-dimensional particle-in-cell simulations, J. Geophys. Res., Space Phys., 118, 2824-2833, doi:10.1002/jgra.50365, 2013.

Chen, C. H. K., Mallet, A., Schekochihin, A. A., Horbury, T. S., Wicks, R. T., and Bale, S. D.: Three-dimensional structure of solar wind turbulence, Astrophys. J., 758, 120, doi:10.1088/0004637X/758/2/120, 2012.

Comişel, H., Verscharen, D., Narita, Y., and Motschmann, U.: Spectral evolution of two-dimensional kinetic plasma turbulence in the wavenumber-frequency domain, Phys. Plasmas, 20, 090701, doi:10.1063/1.4820936, 2013.

Elmegreen, B. G. and Scalo, J.: Interstellar turbulence I: Observations and processes, Ann. Rev. Astron. Astrophys., 42, 211-273, doi:10.1146/annurev.astro.41.011802.094859, 2004.

Escoubet, C. P., Fehringer, M., and Goldstein, M.: The Cluster mission, Ann. Geophys., 19, 1197-1200, doi:10.5194/angeo-191197-2001, 2001.

Gary, S. P., Chang, O., and Wang, J.: Forward cascade of whistler turbulence: Three-dimensional particle-in-cell simulations, Astrophys. J., 755, 142, doi:10.1088/0004-637X/755/2/142, 2012.

He, G.-W. and Zhang, J.-B.: Elliptic model for space-time correlation in turbulent shear flows, Phys. Rev. E, 73, 055303R, doi:10.1103/PhysRevE.73.055303, 2006.

Howes, G. G., Tenbarge, J. M., Dorland, W., Quataert, E., Schekochihin, A. A., Numata, R., and Tatsuno, T.: 
Gyrokinetic simulations of solar wind turbulence from ion to electron scales, Phys. Rev. Lett., 107, 035004, doi:10.1103/PhysRevLett.107.035004, 2011.

Kolmogorov, A. N.: The local structure of turbulence in incompressible viscous fluid for very large Reynolds number, Dokl. Akad. Nauk. SSSR, 30, 299-303, 1941 (Reprinted in Proc. Roy. Soc. A 434, 9-13, doi:10.1098/rspa.1991.0075, 1991).

Kraichnan, R. H.: Kolmogorov's hypothesis and Eulerian turbulence theory, Phys. Fluids, 7, 1723-1734, 1964.

Julien, K. and Knobloch, E.: Magnetorotational instability: recent developments, Phil. Trans. R. Soc. A, 368, 1607-1633, doi:10.1098/rsta.2009.0251, 2010.

Matthaeus, W. H., Goldstein, M. L., and Roberts, D. A.: Evidence for the presence of quasi-two-dimensional nearly incompressible fluctuations in the solar wind, J. Geophys. Res., 95, 20, 673-683, doi:10.1029/JA095iA12p20673, 1990

Matthaeus, W. H., Gosh, S., Ougton, S., and Roberts, D. A.: Anisotropic three-dimensional MHD turbulence, J. Geophys. Res., 101, 7619-7630, doi:10.1029/95JA03830, 1996.

Matthaeus, W. H. and Ghosh, S.: Spectral decomposition of solar wind turbulence: Three-component model, AIP Conf. Proc., The solar wind nine conference, 5-9 October 1998, 471, 519-522, doi:10.1063/1.58688, 1999

Narita, Y. and Glassmeier, K.-H.: Spatial aliasing and distortion of energy distribution in the wave vector domain under multi-spacecraft measurements, Ann. Geophys., 27, 3031-3042, doi:10.5194/angeo-27-3031-2009, 2009.

Narita, Y., Glassmeier, K.-H., Sahraoui, F., and Goldstein, M. L.: Wave-vector dependence of magnetic-turbulence spectra in the solar wind, Phys. Rev. Lett., 104, 171101, doi:10.1103/PhysRevLett.104.171101, 2010.

Narita, Y., Glassmeier, K.-H., and Motschmann, U.: Highresolution wave number spectrum using multi-point measurements in space - The Multi-point Signal Resonator (MSR) technique, Ann. Geophys., 29, 351-360, doi:10.5194/angeo-29-3512011, 2011a.

Narita, Y., Glassmeier, K.-H., Goldstein, M. L., Motschmann, U., and Sahraoui, F.: Three-dimensional spatial structures of solar wind turbulence from $10000-\mathrm{km}$ to $100-\mathrm{km}$ scales, Ann. Geophys., 29, 1731-1738, doi:10.5194/angeo-29-1731-2011, $2011 \mathrm{~b}$.

Petrosyan, A., Balogh, A., Goldstein, M. L., Léorat, J., Marsch, E., Petrovay, K., Roberts, B., von Steiger, R., and Vial, J. C.: Turbulence in the solar atmosphere and solar wind, Space Sci. Rev., 156, 135-238, doi:10.1007/s11214-010-9694-3, 2010.

Rème, H., Aoustin, C., Bosqued, J. M., Dandouras, I., Lavraud, B., Sauvaud, J. A., Barthe, A., Bouyssou, J., Camus, Th., Coeur-Joly, O., Cros, A., Cuvilo, J., Ducay, F., Garbarowitz, Y., Medale, J. L., Penou, E., Perrier, H., Romefort, D., Rouzaud, J., Vallat, C., Alcaydè, D., Jacquey, C., Mazelle, C., d'Uston, C., Möbius, E., Kistler, L. M., Crocker, K., Granoff, M., Mouikis, C., Popecki, M., Vosbury, M., Klecker, B., Hovestadt, D., Kucharek, H., Kuenneth, E., Paschmann, G., Scholer, M., Sckopke, N., Seidenschwang, E., Carlson, C. W., Curtis, D. W., Ingraham, C., Lin, R. P., McFadden, J. P., Parks, G. K., Phan, T., Formisano, V., Amata, E., Bavassano-Cattaneo, M. B., Baldetti, P., Bruno, R., Chionchio, G., Di Lellis, A., Marcucci, M. F., Pallocchia, G., Korth, A., Daly, P. W., Graeve, B., Rosenbauer, H., Vasyliunas, V., McCarthy, M., Wilber, M., Eliasson, L., Lundin, R., Olsen, S., Shelley, E. G., Fuselier, S., Ghielmetti, A. G., Lennartsson, W., Es- coubet, C. P., Balsiger, H., Friedel, R., Cao, J.-B., Kovrazhkin, R. A., Papamastorakis, I., Pellat, R., Scudder, J., and Sonnerup, B.: First multispacecraft ion measurements in and near the Earth's magnetosphere with the identical Cluster ion spectrometry (CIS) experiment, Ann. Geophys., 19, 1303-1354, doi:10.5194/angeo19-1303-2001, 2001.

Sahraoui, F., Belmont, G., Goldstein M., and Rezeau, L.: Limitations of multi-spacecraft data techniques in measuring wavenumber spectra of space plasma turbulence, J. Geophys. Res., 115, A04206, doi:10.1029/2009JA014724, 2010.

Scalo, J. and Elmegreen, B. G.: Interstellar turbulence II: Implications and effects, Ann. Rev. Astron. Astrophys., 42, 275-316, doi:10.1146/annurev.astro.42.120403.143327, 2004.

Schmidt, R. O.: Multiple emitter location and signal parameter estimation, IEEE Trans. Ant., Prop., AP-34, 276-280, 1986.

Servidio, S., Carbone, V., Dmitruk, P., and Matthaeus, W. H.: Time decorrelation in isotropic magnetohydrodynamic turbulence, Europhys. Lett., 96, 55003, doi:10.1209/0295-5075/96/55003, 2011.

Shebalin, J. V., Matthaeus, W. H., and Montgomery, D.: Anisotropy in MHD turbulence due to a mean magnetic field, J. Plasma Phys, 29, 525-547, doi:10.1017/S0022377800000933, 1983.

Taylor, G. I.: The spectrum of turbulence, Proc. R. Soc. Lond. A 164, 476-490, doi:10.1098/rspa.1938.0032, 1938.

Tu, C.-Y., and Marsch, E.: MHD structures, waves and turbulence in the solar wind: Observations and theories, Space Sci. Rev., 73, 1-210, doi:10.1007/BF00748891, 1995.

Turner, A. J., Gogoberidze, G., Chapman, S. C., Hnat, B., and Müller, W.-C.: Nonaxisymmetric anisotropy of solar wind turbulence, Phys. Rev. Lett., 107, 095002, doi:10.1103/PhysRevLett.107.095002, 2011.

Valentini, F., Califano,F., and Veltri, P.: Two-dimensional kinetic turbulence in the solar wind, Phys. Rev. Lett. 104, 205002, doi:10.1103/PhysRevLett.104.205002, 2010.

Verscharen, D., Marsch, E., Motschmann, U., and Müller, J.: Kinetic cascade beyond magnetohydrodynamics of solar wind turbulence in two-dimensional hybrid simulations, Phys. Plasmas, 19, 022305 doi:10.1063/1.3682960, 2012.

von Stein, R., Glassmeier, K.-H., and Dunlop, M.: A configuration parameter for the Cluster satellites, Tech. Rep. 2/1992, Institut für Geophysik und Meteorologie der Technischen Universität Braunschweig, 1992.

Wilczek, M. and Narita, Y.: Wavenumber-frequency spectrum for turbulence from a random sweeping hypothesis with mean flow, Phys. Rev. E, 86, 066308, doi:10.1103/PhysRevE.86.066308, 2012.

Zhao, X. and He, G.-W.: Space-time correlations of fluctuating velocities in turbulent shear flows, Phys. Rev. E, 79, 046316, doi:10.1103/PhysRevE.79.046316, 2009.

Zimbardo, G., Greco, A., Sorriso-Valvo, L., Perri, S., Vörös, Z., Aburjania, G., Chargazia, K., and Alexandrova, O.: Magnetic turbulence in the geospace environment, Space Sci. Rev., 156, 89-134, doi:10.1007/s11214-010-9692-5, 2010. 